\title{
Cryptosporidium cuniculus - new records in human and kangaroo in Australia
}

\author{
Anson V Koehler ${ }^{1}$, Margaret J Whipp ${ }^{2}$, Shane R Haydon ${ }^{3}$ and Robin B Gasser ${ }^{1 *}$
}

\begin{abstract}
Background: To date, Cryptosporidium cuniculus has been found exclusively in rabbits and humans. The present study provides the first published molecular evidence for $C$. cuniculus in an Australian human patient as well as a kangaroo.

Findings: Using PCR-based sequencing of regions in the actin, $60 \mathrm{kDa}$ glycoprotein (gp60) and small subunit of ribosomal RNA (SSU) genes, we identified a new and unique C. cuniculus genotype (akin to VbA25) from a human, and C. cuniculus genotype VbA26 from an Eastern grey kangaroo (Macropus giganteus) in Australia.
\end{abstract}

Conclusions: The characterisation of these genotypes raises questions as to their potential to infect humans and/or other animals in Australia, given that C. cuniculus has been reported to cause cryptosporidiosis outbreaks in Europe.

Keywords: Cryptosporidium cuniculus, Australia, Human, Kangaroo, Novel genotype

\section{Background}

Cryptosporidium is a genus of apicomplexan protozoans that infect humans and other animal hosts, and frequently cause enteritis and associated diarrhoea [1]. Most infections of humans are attributed to C. hominis and C. parvum, but C. canis, C. felis and C. meleagridis, which usually infect dogs, cats and birds, respectively, are also zoonotic [2]. Interestingly, C. cuniculus, usually a parasite of rabbits, is genetically closely related to $C$. hominis and C. parvum, and has been responsible for outbreaks of cryptosporidiosis in humans in the UK [3-5]. In the first recorded outbreak, the infection source was water from a tank contaminated by C. cuniculus-infected European rabbit (Oryctolagus cuniculus), and the protist was identified with the aid of molecular tools [6].

Using the sequences of small subunit of ribosomal RNA (SSU), actin and/or Cryptosporidium oocyst wall protein (COWP) genes, C. cuniculus can be distinguished genetically from the closely related species $C$. hominis. In addition, $C$. cuniculus can be readily identified based on the sequence of the $60 \mathrm{kDa}$ glycoprotein (gp60) gene. Using this locus, two distinct clades, designated Va and $\mathrm{Vb}$, can be classified according to the number of TCA

\footnotetext{
* Correspondence: robinbg@unimelb.edu.au

${ }^{1}$ Faculty of Veterinary and Agricultural Science, The University of Melbourne, Parkville, Victoria 3010, Australia

Full list of author information is available at the end of the article
}

(serine)-tandem repeats in the microsatellite region (e.g., VaA26 = clade Va, with 26 TCA repeats) [3]. Although there is no evidence to suggest that either clade is strictly host-specific, most cases described in humans relate to clade Va [7].

Since the first UK outbreak [3], there has been increasing interest in C. cuniculus due to the zoonotic threat that it poses [6], and this pathogen has been identified in humans and/or rabbits mainly in the UK and China [3,7-10], with some reports from other countries including the Czech Republic, France and Nigeria [11-13]. In addition, a recent outbreak was associated with the deaths of 300 rabbits in Poland [14]. Although C. cuniculus has been recorded in rabbits in south-eastern Australia $[15,16]$, there is no published report of this pathogen from humans or other host species in Australia. Here, we report C. cuniculus genotypes originating from an Australian human patient and, for the first time, from an Eastern grey kangaroo (Macropus giganteus).

\section{Methods}

A faecal sample (collected in 2009) from a human known to be clinically affected by cryptosporidiosis was provided to us (anonymously) by the Microbiology Diagnostic Unit of the Public Health Laboratory (MDU/PHL) of the University of Melbourne. Faecal DNA was isolated following treatment with $10 \%$ polyvinyl-polypyrrolidone, 
using the standard QIAamp DNA Mini Kit (Qiagen, Germany) protocol. In addition, a faecal sample from $M$. giganteus was collected during a surveillance campaign of waterborne pathogens in wildlife within the drinking water catchment areas of Melbourne Water (www.melbournewater.com.au) in Victoria, Australia [16]. This faecal sample was collected in August 2013 in the Yan Yean catchment $(-37.619704 ; 144.901997)$ [16]; the species of kangaroo host was initially established by sight, according to Triggs [17], and then confirmed using an established PCR-based method (unpublished) for the sequencing of a mitochondrial cytochrome oxidase $b$ gene region of $M$. giganteus from faecal DNA isolated using a kit (PowerSoil, Mo-Bio, California). Subsequently, regions of the actin, gp60 (two regions) and/or SSU genes of Cryptosporidium were amplified individually from each of the two faecal DNA samples by PCR and then sequenced as described previously [18]. Nucleotide sequences were deposited in the GenBank database [GenBank: KM366138-KM366142].

\section{Findings}

C. cuniculus was identified in the human faecal DNA sample based on the sequences of amplicons derived from the SSU [GenBank: KM366141] (252 bp), actin [GenBank: KM366138] (782 bp) and gp60 [GenBank: KM366139] (243 bp) gene regions; no sequence differences were detected in the SSU or actin regions compared with published sequences for C. cuniculus [GenBank: EU437413 and GU327783]. Based on the tandem repeat region in gp60, the parasite detected was classified as C. cuniculus VbA25. Of interest was a 36 bp deletion determined by comparison with a gp60 reference sequence [GenBank: KM366139] (Figure 1). This deletion was confirmed by independent sequencing of this region using two independent sets of primers to gp60 and did not result in a frame shift in the coding region (c.f. Figure 1). With the exception of this deletion and a single nucleotide substitution $(\mathrm{G} \rightarrow \mathrm{A})$ (Figure 1), the gp60 sequence (representing $\mathrm{VbA25}$ ) was the same as that of a reference sequence [GenBank: GU971647] from a human clinical case in the UK [8]; it was also $98 \%$ similar to another C. cuniculus sequence from a rabbit in Australia [GenBank: KC283003] (also classified as VbA25), but contained an additional 'ACA' repeat following the characteristic serine tandem repeat region.

C. cuniculus was also identified in the faecal sample from a $M$. giganteus individual from the Yan Yean catchment based on the SSU [GenBank: KM366142] (557 bp) and gp60 [GenBank: KM366140] (282 bp) sequences from amplicons produced. The gp60 sequence (VbA26) was similar to that from C. cuniculus (VbA25; with an additional serine repeat) [GenBank: KC283003] from the same catchment (2011), and identical to sequences [GenBank: HM852433 and KC283004] originating from C. cuniculus from the same lagomorph species from another Melbourne Water catchment (Sugar Loaf; -37.681999; 145.293841) in 2010.

To date, C. cuniculus has been found exclusively in rabbits and humans $[6,7,14]$. Here, we provide molecular evidence for a new $C$. cuniculus genotype in a human in Australia and, for the first time, for the occurrence of $C$. cuniculus in a kangaroo. In Victoria, Australia, as part of an ongoing pathogen detection program, in which we have molecularly tested $>1500$ faecal samples from kangaroos and wallabies ([16]; Koehler et al. unpublished data), this is the first instance of C. cuniculus being detected in the faeces from any macropodid host. The presence of $C$. cuniculus DNA in the faecal sample from the $M$. giganteus individual indicates, but does not prove, that this macropod was infected with this pathogen. There is a remote possibility that C. cuniculus oocysts passed in faeces related to pseudoparasitism [19], as a result of the kangaroo ingesting such oocysts while grazing on grass contaminated by faeces from infected rabbits. Nonetheless, given that many kangaroos graze in some of Melbourne's water catchment areas, where rabbits are common, we would have expected to identify C. cuniculus much more frequently by molecular testing than one of $>1500$ samples tested to date. Therefore, the probability that this $M$. giganteus individual harboured a cryptosporidial infection is considered high.

The zoonotic risk of $C$. cuniculus to human health is clearly evidenced by the first outbreak in the UK in 2008, in which 29 people reported illness after drinking water contaminated with $C$. cuniculus from a rabbit [5]. In

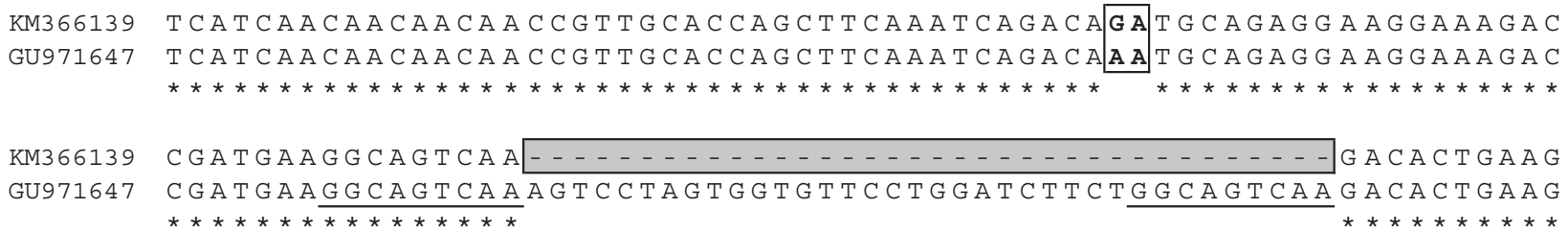

Figure 1 Sequence alignment. An alignment of the sequence of part of the gp60 gene (derived from a Cryptosporidium cuniculus genotypes from a human) [GenBank: KM366139] with its closest sequence match in the GenBank database [GenBank: GU971647] (C. cuniculus from human). Bold-type indicates a variable region of two bases. The deletion is indicated in grey (36 positions). The repeat regions before and after the deletion are underlined. Identical nucleotides are indicated with an asterisk. 
addition, recently, the dramatic effect of $C$. cuniculus on its lagomorph host was demonstrated when 300 rabbits were reported to have died from cryptosporidiosis linked to this species of Cryptosporidium (c.f. [14]). The occurrence of C. cuniculus genotypes in rabbits [15] and, here, in $M$. giganteus suggests that such genotypes might be able to spread to other native mammals and/or humans in Australia. Therefore, there is a need to diligently monitor Cryptosporidium in the vicinity of drinking water catchments (c.f. [16]) and in drinking water. The need for monitoring by molecular tools is particularly crucial in Victoria and other states of Australia, where rabbit populations have flourished ever since their introduction in 1859, causing a widespread degradation of natural ecosystems [20]. Such monitoring is also critical, given that people in Victoria consume unfiltered drinking water from natural catchment areas, and that currently used water treatment processes/procedures do not destroy oocysts of Cryptosporidium [21].

\section{Conclusions}

Sequencing of regions in the actin, gp60 and SSU genes has led to the identification of a new and unique $C$. cuniculus genotype (similar to VbA25 from a human); for the first time, C. cuniculus (genotype VbA26) has been found in an Eastern grey kangaroo (M. giganteus) in Australia. The characterisation of this and similar genotypes raises questions as to their potential to infect humans and/or other animals in Australia, given that C. cuniculus has been reported to cause cryptosporidiosis outbreaks in Europe.

\section{Competing interests}

The authors declare that they have no competing interests.

\section{Authors' contributions}

AK carried out the molecular work and drafted the manuscript. MW supplied the samples. SH participated in the coordination of the study. RG participated in the design, analysis and drafting of the manuscript. All authors read and approved the final version of the manuscript.

\section{Acknowledgements}

Funds from the Australian Research Council (ARC), the National Health and Medical Research Council (NHMRC) and Melbourne Water Corporation are gratefully acknowledged.

\section{Author details}

${ }^{1}$ Faculty of Veterinary and Agricultural Science, The University of Melbourne Parkville, Victoria 3010, Australia. ${ }^{2}$ Microbiological Diagnostic Unit, Public Health Laboratory, Doherty Institute, The University of Melbourne, Parkville, Victoria 3010, Australia. ${ }^{3}$ Melbourne Water Corporation, Docklands, Victoria 3008, Australia.

Received: 2 September 2014 Accepted: 20 October 2014

Published online: 30 October 2014

\section{References}

1. Xiao L, Fayer R: Molecular characterisation of species and genotypes of Cryptosporidium and Giardia and assessment of zoonotic transmission. Int J Parasitol 2008, 38:1239-1255.

2. Xiao L, Feng Y: Zoonotic cryptosporidiosis. FEMS Immunol Med Microbiol 2008, 52:309-323.
3. Chalmers RM, Robinson G, Elwin K, Hadfield SJ, Xiao L, Ryan UM, Modha D, Mallaghan C: Cryptosporidium rabbit genotype, a newly identified human pathogen. Emerg Infect Dis 2009, 15:829-830.

4. Robinson G, Wright S, Elwin K, Hadfield SJ, Katzer F, Bartley PM, Hunter PR, Nath M, Innes EA, Chalmers RM: Re-description of Cryptosporidium cuniculus Inman and Takeuchi, 1979 (Apicomplexa: Cryptosporidiidae): morphology, biology and phylogeny. Int J Parasitol 2010, 40:1539-1548.

5. Puleston RL, Mallaghan CM, Modha DE, Hunter PR, Nguyen-Van-Tam JS, Regan CM, Nichols GL, Chalmers RM: The first recorded outbreak of cryptosporidiosis due to Cryptosporidium cuniculus (formerly rabbit genotype), following a water quality incident. J Water Health 2014, 12:41-50.

6. Robinson G, Chalmers R: The European rabbit (Oryctolagus cuniculus), a source of zoonotic cryptosporidiosis. Zoonoses Public Health 2010, 57:e1-e13.

7. Zhang W, Shen Y, Wang R, Liu A, Ling H, Li Y, Cao J, Zhang X, Shu J, Zhang L: Cryptosporidium cuniculus and Giardia duodenalis in rabbits: genetic diversity and possible zoonotic transmission. PLOS ONE 2012, 7:e31262.

8. Chalmers RM, Elwin K, Hadfield SJ, Robinson G: Sporadic human cryptosporidiosis caused by Cryptosporidium cuniculus, United Kingdom, 2007-2008. Emerg Infect Dis 2011, 17:536-538.

9. Shi K, Jian F, Lv C, Ning C, Zhang L, Ren X, Dearen TK, Li N, Qi M, Xiao L: Prevalence, genetic characteristics, and zoonotic potential of Cryptosporidium species causing infections in farm rabbits in China. J Clin Microbiol 2010, 48:3263-3266.

10. Liu X, Zhou X, Zhong Z, Chen W, Deng J, Niu L, Wang Q: New subtype of Cryptosporidium cuniculus isolated from rabbits by sequencing the $g p 60$ gene. J Parasitol 2014, 100:532-536.

11. Ryan UM, Xiao L, Read C, Zhou L, Lal AA, Pavlasek I: Identification of novel Cryptosporidium genotypes from the Czech Republic. Appl Environ Microbiol 2003, 69:4302-4307.

12. Molloy SF, Smith HV, Kirwan P, Nichols RA, Asaolu SO, Connelly L, Holland $\mathrm{CV}$ : Identification of a high diversity of Cryptosporidium species genotypes and subtypes in a pediatric population in Nigeria. Am J Trop Med Hyg 2010, 82:608-613.

13. ANOFEL Cryptosporidium National Network: Laboratory-based surveillance for Cryptosporidium in France, 2006-2009. Euro Surveillance 2010, 15:19642.

14. Kaupke A, Kwit E, Chalmers R, Michalski M, Rzeżutka A: An outbreak of massive mortality among farm rabbits associated with Cryptosporidium infection. Res Vet Sci 2014, 97:85-87.

15. Nolan MJ, Jex AR, Haydon SR, Stevens MA, Gasser RB: Molecular detection of Cryptosporidium cuniculus in rabbits in Australia. Infect Genet Evol 2010, 10:1179-1187.

16. Nolan MJ, Jex AR, Koehler A, Haydon SR, Stevens MA, Gasser RB: Molecular-based investigation of Cryptosporidium and Giardia from animals in water catchments in southeastern Australia. Water Res 2013, 47:1726-1740

17. Triggs B: Tracks, Scats and Other Traces: a Field Guide to Australian Mammals. South Melbourne: Oxford University Press; 2004.

18. Koehler AV, Whipp M, Hogg G, Haydon SR, Stevens MA, Jex AR, Gasser RB: First genetic analysis of Cryptosporidium from humans from Tasmania, and identification of a new genotype from a traveller to Bali. Electrophoresis 2014, 35:2600-2607.

19. Bowman DD, Georgi JR: Georgis' Parasitology for Veterinarians. Saunders/ Elsevier: St. Louis; 2009.

20. Cooke BD: Rabbits: manageable environmental pests or participants in new Australian ecosystems? Wildl Res 2012, 39:279-289.

21. Yoder JS, Beach MJ: Cryptosporidium surveillance and risk factors in the United States. Exp Parasitol 2010, 124:31-39.

doi:10.1186/s13071-014-0492-8

Cite this article as: Koehler et al:: Cryptosporidium cuniculus - new records in human and kangaroo in Australia. Parasites \& Vectors 2014 7:492. 